\title{
BMJ Open The role of a decision-support smartphone application in enhancing community health volunteers' effectiveness to improve maternal and newborn outcomes in Nairobi, Kenya: quasi-experimental research protocol
}

Pauline Bakibinga, Eva Kamande, Milka Omuya, Abdhalah K Ziraba, Catherine Kyobutungi

To cite: Bakibinga $P$, Kamande E, Omuya M, et al. The role of a decision-support smartphone application in enhancing community health volunteers' effectiveness to improve maternal and newborn outcomes in Nairobi, Kenya: quasi-experimental research protocol. BMJ Open 2017;7:e014896. doi:10.1136/ bmjopen-2016-014896

- Prepublication history and additional material are available. To view, please visit the journal online (http://dx.doi.org/10. 1136/bmjopen-2016-014896).

Received 25 0ctober 2016 Revised 5 May 2017 Accepted 19 May 2017

\section{CrossMark}

Health Challenges and Systems Research Program, African Population and Health Research Center, Nairobi, Kenya

Correspondence to Dr Pauline Bakibinga; paulabak80@gmail.com

\section{ABSTRACT}

Introduction Improving maternal and newborn survival remains major aspirations for many countries in the Global South. Slum settlements, a result of rapid urbanisation in many developing countries including Kenya, exhibit high levels of maternal and neonatal mortality. There are limited referral mechanisms for sick neonates and their mothers from the community to healthcare facilities with ability to provide adequate care. In this study, we specifically plan to develop and assess the added value of having community health volunteers (CHVs) use smartphones to identify and track mothers and children in a bid to reduce pregnancyrelated complications and newborn deaths in the urban slums of Kamukunji subcounty in Nairobi, Kenya. Methods and analysis This is a quasi-experimental study. We are implementing an innovative, mHealth application known as mobile Partnership for Maternal, Newborn and Child Health (mPAMANECH) which uses dynamic mobile phone and web-portal solutions to enable CHVs make timely decisions on the best course of action in their management of mothers and newborns at community level. The application is based on existing guidelines and protocols in use by CHVs. Currently, CHVs conduct weekly home visits and make decisions from memory or using unwieldy manual tools, and thus prone to making errors. mPAMANECH has an in-built algorithm that makes it easier, faster and more likely for CHVs to make the right management decision. We are working with a network of selected CHVs and maternity centres to pilot test the tool. To measure the impact of the intervention, baseline and end-line surveys will be conducted. Data will be obtained through qualitative and quantitative methods.

Ethics and dissemination Ethical approval for the study was obtained from the African Medical Research Foundation. Key messages from the results will be packaged and disseminated through meetings, conference presentations, reports, fact sheets and academic publications to facilitate uptake by policymakers.
Strengths and limitations of this study

- The project seeks to address a critical challenge in the healthcare system in a slum context: identification and referral of ill mothers and newborns.

- It uses the existing community health volunteer (CHV) work tools and mobile phone technology to show how an evidence-based intervention can improve health outcomes at community level.

- A potential harmful unintended consequence stems from the fact that CHVs are not medically trained and as such could make a decision to treat at home rather than refer (even when the system advises otherwise) with possible negative health consequences for the mothers and their newborns.

- However, since the system allows health workers to access these data, they will be in charge of following up on such cases to avoid unnecessary delays and such unintended consequences.

\section{INTRODUCTION}

Annually, close to 300000 women die from maternal causes, 3.3 million children are stillborn while an additional 3.3 million die within the first month of life. ${ }^{1-3}$ A vast majority of these deaths occur in sub-Saharan Africa and South Asia. Most maternal and newborn deaths are due to causes directly related to pregnancy and childbirth. ${ }^{124}$ Most of these causes are preventable with existing cost-effective interventions. Kenya has made some progress in improving maternal and newborn survival over the years. Maternal mortality has progressively reduced from 488/100 000 to $362 / 100000$ between $2008 / 2009$ and 2014. Neonatal mortality has reduced from 31 to 22 deaths per 1000 live births, between $2008 / 2009$ and 2014. ${ }^{5} 6$ Neonatal deaths 
account for about $60 \%$ of all infant deaths in Kenya. ${ }^{6}$ Despite the existence and knowledge about simple strategies and techniques to reduce maternal and newborn deaths, these interventions are not up to scale due to inadequacy of healthcare facilities, trained personnel, information, poor referral systems and pervasive poverty.

\section{Community health volunteers}

The need to ensure universal health coverage amidst a global healthcare workers crisis saw the identification of community health volunteers (CHVs) as a critical alternative and workforce at the community level. ${ }^{7} \mathrm{CHV}$ are volunteers identified from communities that they reside in and offered a few days' basic training based on a set curriculum before they are assigned community health promotion roles. CHVs belong to established community units with a responsibility of making weekly home visits to households within designated geographical areas. CHVs were identified under Kenya's Community Health Strategy as a means of alleviating the healthcare worker crisis. Under the strategy, CHVs are supervised by community health extension workers (CHEWs). To date, CHVs continue to bridge the critical gap between communities and the formal health system, and many positive lessons have been learnt in Kenya and other parts of the world. ${ }^{8-10}$ CHVs in Kenya, like in many emerging economies, are responsible for the majority of grass roots healthcare delivery, and as such, continue to play a very critical role in primary healthcare delivery.

\section{Decision-support systems}

Clinical decision support (CDS) refer to tools used to improve decision-making in the patient care workflow. ${ }^{11}$ CDS provides clinicians, staff, patients or other individuals with knowledge and person-specific information, intelligently filtered or presented at appropriate times, to enhance health and healthcare. ${ }^{12} 13$ These tools include computerised alerts and reminders to care providers and patients, clinical guidelines, condition-specific order sets, focused patient data reports and summaries, documentation templates, diagnostic support and contextually relevant reference information, among other tools. ${ }^{14}$ They are beneficial in reducing errors, increasing quality of care and improving efficiency.

\section{Mobile technology for health}

Mobile technologies have the potential to bridge systemic gaps needed to improve access to and use of health services, particularly among underserved populations. ${ }^{15}$ Worldwide, the use of mobile and/or electronic devices to support both medical and public health practice and research (mHealth) is increasingly being appreciated. ${ }^{16-19}$ The high and ever-growing mobile penetration coupled with investments from technology companies that provide accessible platforms onto which innovations can establish and offer value-based products. ${ }^{17}$ Innovations include mobile phone platforms/applications and microinsurance packages. Given the nature and tight time frames of most obstetric and neonatal emergencies, which more often than not demand quick access to quality care at short notice, mobile phone innovations are a cheap and fast solution especially in areas with poor healthcare infrastructure which have shown improvements in maternal and child health outcomes. ${ }^{19} 20$ The majority of innovations on the market are tailored towards promoting reproductive, maternal and child health. ${ }^{16}{ }^{21}$ In addition, various projects have been designed and evaluated on CHVs use of mobile technology to deliver interventions. ${ }^{22}{ }^{23}$ Decision-support systems have been highlighted as a critical factor in reducing errors in evidence-based clinical practice. For clinicians, studies have demonstrated that computerised decision-support systems can be used to improve compliance to practice guidelines. ${ }^{12} 13$ Given the limited training obtained and skills that the CHVs possess, coupled with the distances they work from the clinical settings, mobile information technology tools enable them to provide much needed health services.

Over the last decade, more mobile and electronic information tools have been developed, tested and implemented with CHVs to support their work roles. The tools help the CHVs in surmounting challenges such as lack of appropriate work tools and inadequate supportive supervision and training. ${ }^{22} 23$ These tools have been instrumental in improving access to care by marginalised population groups subjected to stigma and those in hard-to-reach areas by reducing both time and cost of travel. As such, research on CHVs' use of mHealth tools is important. Several pilot projects, using multiple designs and measures have been implemented. The projects have reported improvements in services rendered by CHVs and the related health outcomes for communities. In sub-Saharan Africa, most of the interventions have demonstrated improvements in the CHVs' delivery of maternal, newborn and child health, tuberculosis and sexual and reproductive health services, among others. ${ }^{20}{ }^{22}$ The innovations largely use short message service (SMS) and hotlines enabling mothers to access vital information and timely referrals as a result of better and faster communication and transport. Available evidence shows the usefulness of mHealth tools in facilitation of compliance with practice guidelines and process improvement. ${ }^{22}$ However, many of the interventions have been limited methodologically by their inability to demonstrate effectiveness and/or impact related to patient outcomes. In addition, a lack of use of nationally accepted guidelines and open-source tools limits the scalability of these interventions. In addition, available literature does not provide cost estimates necessary for delivery of programme targets. Furthermore, a top to bottom approach that does not incorporate the ideas of the CHVs in the development of the tools is a major impediment as successful projects use the CHVs as experts in the design and management of their interventions. ${ }^{22}$

The African Population and Health Research Center (APHRC) has implemented a health systems strengthening project, Partnership for Maternal, Newborn and 
Child Health (PAMANECH) in Korogocho and Viwandani informal settlements of Nairobi, Kenya. ${ }^{24}$ The project sought to strengthen the healthcare delivery system in the urban informal settlements to be more responsive to the health needs of mothers and their children through enhanced service delivery public-private partnerships. In a bid to improve the health management information system, an innovative mHealth application, was developed. The mobile PAMANECH (mPAMANECH) application was developed as an integrated data capture tool running as a mobile app with selected reporting forms for CHVs in Kenya. It operates in an interconnected network of CHVs and health facilities within a defined local system. It is designed to replace the numerous paper-based forms that do not allow integration of patient data from the community to the health facility and back for better referral and management of patients. This system has been seen to improve the reporting abilities of CHVs as it is less cumbersome than the paper-based system and enhanced data quality as it has a function that limits saving data until all necessary fields are filled in. ${ }^{25}$ In addition, the CHEW can access a CHV's data remotely without having to wait for the end of the month for the CHV summaries. The desire to improve the functionality of the existing system to include a decision-support function provides the basis on the new project.

This project attempts to bridge this gap by incorporating CHV views, using the tools approved by the Ministry of Health and allowing for assessment of health outcomes in the design and implementation of the project. The decision-support system is developed from open-source tools. In addition, a cost-effectiveness analysis would be conducted to inform county and national implementation plans regarding the necessary investments for set-up and maintenance.

\section{METHODS AND ANALYSIS \\ Research objectives}

The overall objective of the proposed work is to develop and validate a decision-support algorithm within an mHealth application in improving maternal and newborn health outcomes in urban slums in Kamukunji subcounty in Nairobi, Kenya. The project seeks to assess the added value of using a CHV decision-support module of mPAMANECH in reducing prenatal and postnatal maternal complications and newborn deaths.

Specifically, the project will assess (1) the feasibility and acceptability of using a decision-support module of mPAMANECH and (2) whether decision-support platform contributes to increase in use of Maternal and Newborn Health (MNH) services. We will measure several outcomes (figure 1) related to the two specific objectives above. The outcome measures have been used in different studies assessing CHV abilities to assess and make referrals. ${ }^{26} 27$ Online supplementary files 1-3 show the quantitative and qualitative measures under study.
Conceptual framework, theory of change and operationalisation

Our innovation centres around enhancing the functionality and utility of the current mPAMANECH application to include a community decision-support tool to be used within a local system comprised CHVs and five maternity centres serving five slum settlements in Nairobi. The decision-support module in the mPAMANECH application will supplement the existing MNCH data capture modules and help in the identification of high-risk facing pregnant women, new mothers and newborns with complications, and to make timely and correct decisions on referral for cases that need intervention. The main beneficiaries are pregnant women (over 24 weeks), mothers in the immediate postpartum period and their newborns (up to 28 days old).

The use of CHVs in delivering the mobile phone-based intervention and follow-up by the sub-County Health Management Team (sCHMT) will ensure sustained support and adherence to the intervention during pregnancy, immediate post partum and in the neonatal period. The mPAMANECH has the official 100, 513, 514 and 515 forms in use by CHVs and CHEWs, who supervise the CHVs. ${ }^{28}{ }^{29}$ A dedicated team of CHEWs will ensure that the intervention is delivered as expected and non-adherence captured and documented. CHV adherence will be measured by the CHEWs who will provide additional support, correcting CHVs but not forms. They will be used to sample some of the CHVs' work. For each woman or newborn with referral symptom, seen at household level, we will compare the CHEW's assessment with that of the CHV in regard to the proportion of cases for whom community health volunteers (CHVs) identified and indicated correctly on their forms as requiring a referral, and the proportion for whom a written referral was provided. This is beneficial because the review meetings are at the end of the month. The sampling of the work will enable ongoing supportive supervision to improve the quality of the CHVs work. In addition, more assessment will be made for those referrals that will be seen at health facility level, comparing danger signs identified by the CHV to those seen by the clinician.

The attributes of the solution include: (1) use of any android devices - the entire application is operable on phones, tablets or desktops/laptops; (2) within a linked local system-messaging is delivered internally via the portal and no extra network charges are required for messaging; (3) the application is bandwidth light and takes about Kenya Shillings 1100 a month per user, based on local data bundle charges. The application can work offline and auto-synchronise as soon as network connectivity is re-established; (4) updates are automated and there is no need for an IT expert to have physical access to the device to execute a software patch or change; (5) the application has security features that protect users' confidentiality and limit access to a closed but linked local system of health facilities and CHVs with varied access rights; (6) it has the ability to limit use of other 


\begin{tabular}{|c|c|}
\hline Domain of Inquiry & Key Outcome Indicators \\
\hline $\begin{array}{l}\text { Assess the feasibility and acceptability of } \\
\text { using a decision-support module of } \\
\text { mPAMANECH }\end{array}$ & $\begin{array}{l}\text { Percentage time for which the system is down on a } \\
\text { monthly basis } \\
\text { Number of community health volunteers effectively using } \\
\text { the decision support tool } \\
\text { Proportion of community health volunteers able to identify } \\
\text { at least } 4 \text { danger signs among neonates } \\
\text { Proportion of community health volunteers able to identify } \\
\text { at least } 1 \text { danger signs among neonates } \\
\text { Proportion of community health volunteers able to identify } \\
\text { at least } 2 \text { post-partum complications } \\
\text { Percentage of correct referral decisions by community } \\
\text { health volunteers for post-partum mothers with } \\
\text { complications } \\
\text { Percentage of correct referral decisions by community } \\
\text { health volunteers for neonates with danger signs } \\
\text { Proportion of pregnant women referred for antenatal care } \\
\text { by community health volunteers } \\
\text { Proportion of mothers referred for postnatal care by } \\
\text { community health volunteers }\end{array}$ \\
\hline $\begin{array}{l}\text { Determine the decision-support platform's } \\
\text { effect on utilization of maternal and newborn } \\
\text { health services }\end{array}$ & $\begin{array}{l}\text { Proportion of women attending at least } 4 \text { antenatal care } \\
\text { Proportion of all mothers attending at least } 2 \text { postnatal care } \\
\text { Proportion of deliveries assisted by trained personnel } \\
\text { Percentage of women who accessed first antenatal care } \\
\text { within the 1st trimester } \\
\text { Proportion of women referred for post-partum family } \\
\text { planning } \\
\text { Proportion of newborns with at least one danger sign } \\
\text { referred by community health volunteers } \\
\text { Proportion of low birth weight newborns referred } \\
\text { Proportion of women lost during follow up } \\
\text { Proportion of newborns lost during follow up }\end{array}$ \\
\hline
\end{tabular}

Figure 1 Key outcome measures. CHV, community health volunteer; mPAMANECH, mobile Partnership for Maternal, Newborn and Child Health.

phone functionality to only allow this application to run. This helps prevent abuse of the device and promote saving on bandwidth. We aim to enhance the functionality and utility of the current mPAMANECH application to include a community decision-support tool to be used within a local system comprised of selected CHVs and five maternity centres serving slum settlements in Nairobi. In the 'Operationalisation' section, we describe more on how the decision-support system is expected to work.

From our proposed theory of change, figure 2, we anticipate that the decision-support module of mPAMANECH will assist the CHVs in the identification of high-risk pregnant women, new mothers and newborns with complications and to make timely and correct decisions on referral for cases that need intervention. With the improved knowledge and skills possessed by the CHVs, more women and neonates in need of healthcare will be identified and referred to the necessary health facilities. As a result, there will be increased use of maternal and neonatal health services and reduced maternal and neonatal complications and deaths.

\section{Operationalisation of the decision-support system}

The mobile decision-support tool/ system (mDST/S) mobile application (app) is an Android app that installs from the phone and runs as an application (figure 3). The app will be hosted at Google store and is accessible for download via the internet automatically to the user handset.

Once installed, the handset and the application will be configured with the credentials of the CHV including username and password, which are linked with the operating phone number. This set-up allows the system to register and associate collected records 


\section{INPUTS}

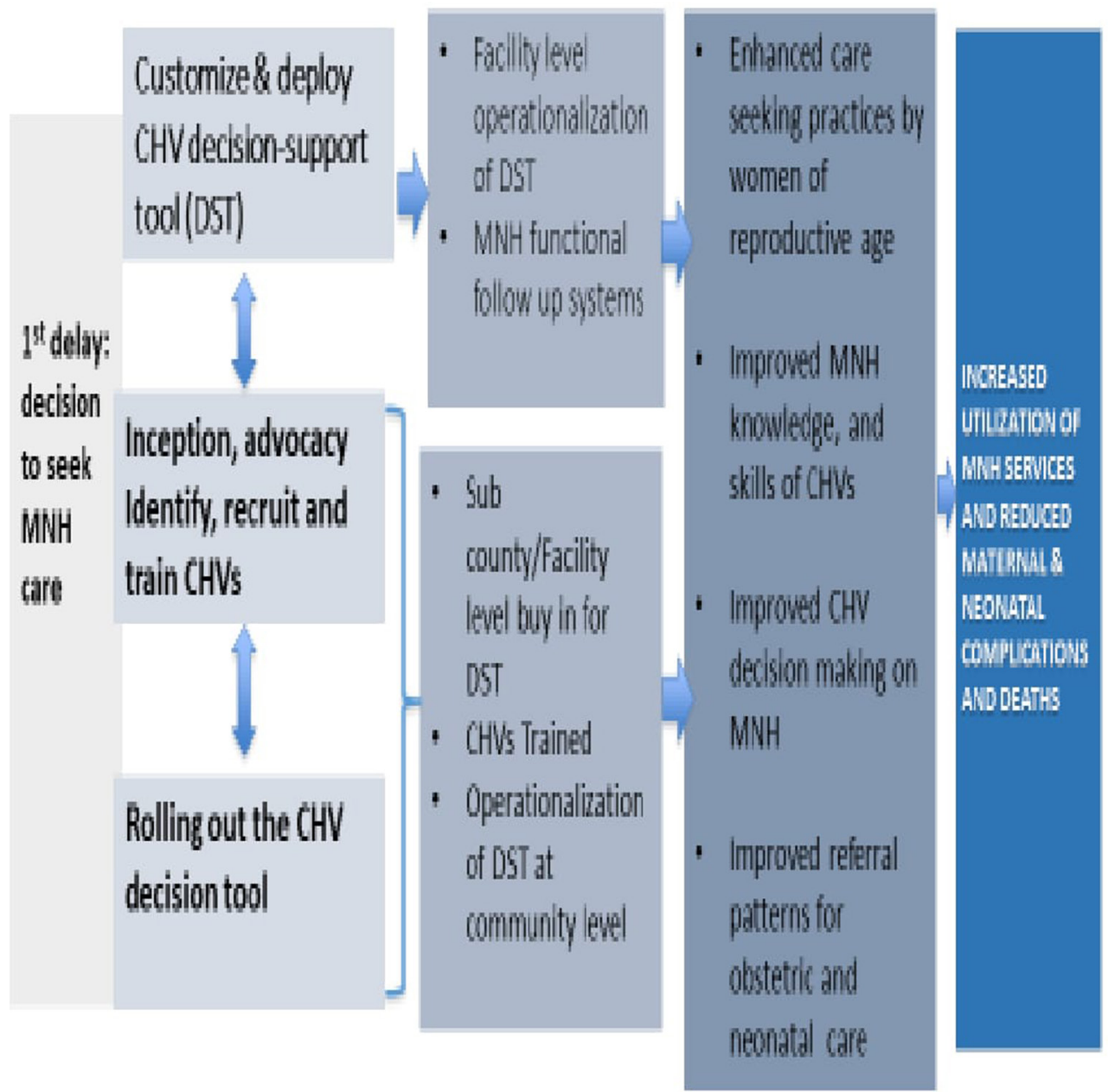

Figure 2 Theory of change. CHV, community health volunteer; DSS, decision-support system.

to the respective CHV; useful for reporting and analysis purposes.

The CHVs will be provided with a mobile phone running the mHealth app for data collection which is then relayed to the head office at APHRC campus. When visiting households for data collection, by initiating a new record the system automatically picks the geolocation of the household and associates it with the record.

The CHV will complete the respective form and save the information at any stage on the handset. This method allows the CHV to continue collecting more information and only submit the completed form to the server over the internet. Where the handset detects that internet connectivity is not available for one reason or the other, the completed forms will be stored in the phone and only be relayed to the server when the connectivity is established. Because the application is in communication with the server in real time, any update of the data is immediately available to the CHVs. The application is preconfigured with danger signs for both mothers and newborns as defined by the WHO. ${ }^{30}$ When any of the danger signs is picked by the system, the CHV is immediately prompted to refer the patient to a facility within the network.

The system is to be set up in such a way that the CHV will not be allowed to proceed and complete the forms until the referral is done. The patient's information will be available to all the facilities within the network and he/she will be able to visit any of the networked facilities for treatment. This information is also relayed to the rest of the other facilities and a record of the visit is kept in the server. Within 24 hours if the patient has not been seen in any of the networked facilities, an SMS is relayed to the respective $\mathrm{CHV}$ handset. At this point, the $\mathrm{CHV}$ should follow up with the patient to inquire about their 


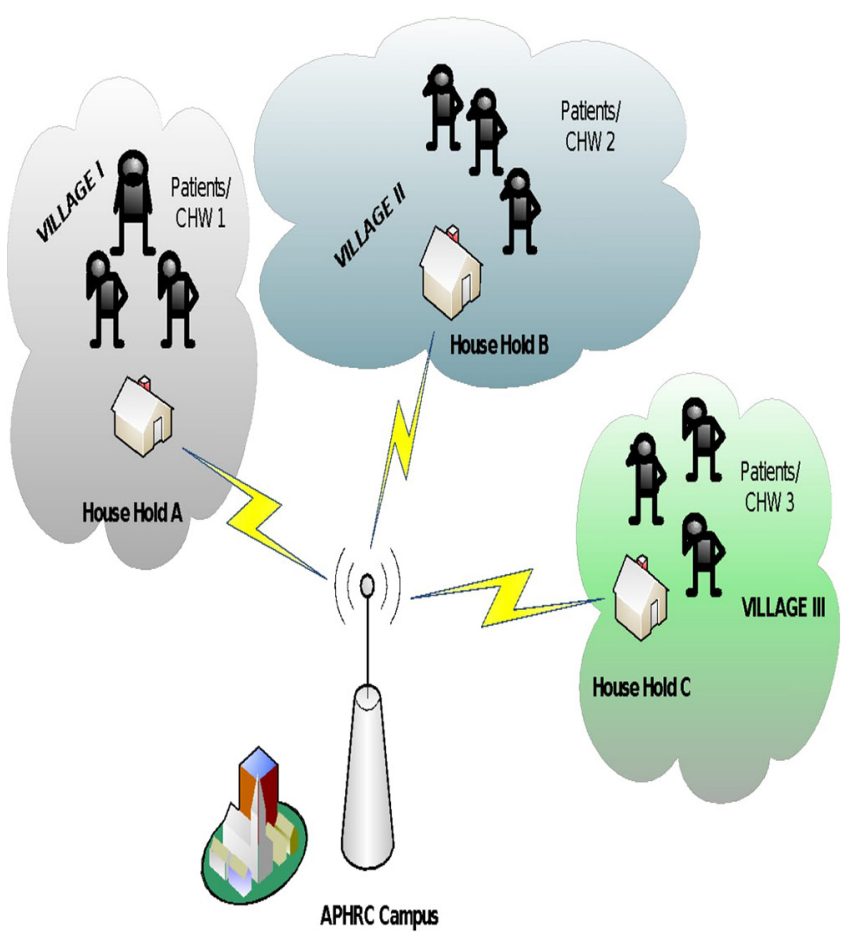

Figure 3 The system. APHRC, African Population and Health Research Center; CHW, community health worker.

condition and whether they chose to honour the referral to a facility outside the network or not.

When the patient visits a facility within the network, using the household number and the name, he/she will be easily identified and treated. The clinician is able to record the treatment in the patient's information, which is recorded in the server.

Both the CHV and the clinician's information are available to a reviewer in real time from the web. The reviewer's role would be to assess the CHV's reason for referral and the clinician's final diagnosis for correctness. The feedback from the reviewer will be available and shared on a monthly basis during review meetings. Every week, the CHEW, will randomly sample referrals made by each CHV to assess their correctness. This will be compared with the 'reviewers' summary.

\section{The mobile Decision Support Tool/System solution-key features}

The key features of mDSS solution are given below and in figure 4.

\section{CHV (mobile app)}

Through this app, the mobile Decison Support Tool/ System (mDST/S) data collection tools are accessible on the CHV mobile handset. It allows for completion and submission of the information to the mDSS Server.

\section{mDST/S server}

A database-driven web application that acts as a host for the collected information. It also has a web interface for data manipulation by the facility healthcare worker(s) and reviewer.

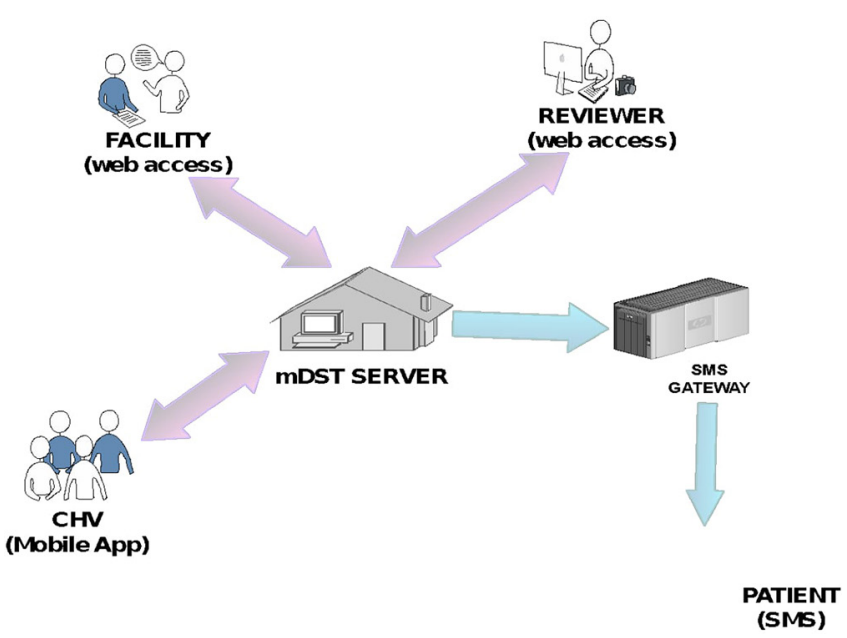

Figure 4 How the system works. CHV, community health volunteer; SMS, short message service, mDST mobile decision support tool

The mobile Decision Support Tool/System (mDST/S) server application incorporates an application logic component that connects with the SMS and email gateways. This allows for real-time communication of certain critical indicators to the respective recipients. For example, where a patient has not shown up at the referred facility, the CHV will be alerted.

Facility (web access)

This module is accessible at the facility via the web. The application allows the clinician to treat the patient and record the treatment. The facility portal is accessible by facility staff to manage referrals. The 'Messages' to alert the CHVs are to be integrated with WhatsApp for effective message delivery over the same mobile phone.

\section{Reviewer (web access)}

This module is also accessible via the web and provides the reviewer a view to assess both the CHV referral report and the clinician's diagnosis. It is based on this analysis that a decision is measured.

\section{Null hypothesis}

Usage of a CHV decision-support module does not have an effect on use of MNH services.

\section{Study design and sampling strategy}

\section{Study design}

To answer the question on effect of the decision-support system, we will use a quasi-experimental design with preassessments and postassessments to determine the impact, if any, on the MNH services and selected health indicators.

We will work with three community units (CUs) from which a group of $50 \mathrm{CHVs}$ will serve as the intervention group. Three CUs in Embakasi subcounty, with $50 \mathrm{CHVs}$ will serve as the control. The CUs in the control group will be geographically distant from the intervention site to limit contamination. The control group will be 
facilitated within the normal standard of practice which includes paper-based reporting. The idea is to compare access with MNH services, referral practices and MNH outcomes between those with a decision-support tool with those working with traditional paper-based tools.

We will conduct a baseline survey and an end line at least 1 year after the full implementation of the intervention. The control group will be assessed at the same time as the surveys are being conducted in the intervention group. To strengthen the case for causal inference, we will also do a systematic monitoring and documentation of the intervention based on our theory of change. The monitoring and documentation will also capture any other contextual factors that may influence the same outcomes as our intervention.

To answer the question on feasibility, we will conduct a mixed-method assessment of the CHVs and CHEWs. Qualitative assessments of their current work experiences with the paper-based system as well as after the introduction of the mobile-based system will be conducted. We will assess ease of use, challenges experienced and opportunities for improvement. These will be triangulated with their Health Management Infomation System (HMIS) reports (paper and electronic).

\section{Study setting}

The intervention site covers informal settlements in Kamukunji subcounty, which, like other slums, are characterised by poverty, poor coverage of social services and poor health outcomes. We will identify CUs and five facilities that serve the CUs. Embakasi subcounty will serve as the control site. We will identify three CUs to serve as controls.

\section{Sampling}

The CUs in the intervention and control groups will be purposively selected based on discussions with the subcounty community strategy coordinators for Kamukunji and Embakasi. We will select CUs with the worst health indicators for both the intervention and control groups, including those serving informal settlements, and with more likelihood to benefit from the intervention.

For the population based surveys the detailed strategies are defined below:

\section{Quantitative study}

\section{Study population}

The project will target households with women of reproductive age (15-49 years) in the informal settlements of Kamukunji and Embakasi. It will also target healthcare providers in five selected health facilities as well as the subcounty Health Management teams of Kamukunji, Embakasi, Makadara and Nairobi City County.

A quantitative survey will be conducted focussing mainly on maternal and newborn health and family planning services.

\section{Sample size estimation}

The key outcome of interest is correct referral practices. Using proportion of neonates with at least one danger sign referred by CHVs as the indicator, since it is representative of the target population, the total sample size for each group has been estimated as 173 (346 in total). Using a difference in proportion $13.3 \%$ for neonates with danger signs referred (rising from $16.7 \%$ to $30 \%$ ), at $95 \%$ CI for $80 \%$ power and sample proportions of $50 \%$ in the intervention and $40 \%$ in the control groups, respectively, we came up with a sample size of 315 . Accounting for a potential non-response rate of $10 \%$ based on previous studies in similar study populations, the effective sample size is 346 . Using correct decisions made by CHVs (one of the indicators to be measured), the sample size estimated as 199. 346 is therefore an appropriate sample size.

\section{Sampling procedure}

To measure feasibility and acceptability, all the CHVs in the intervention site will be assessed, in addition to an audit of the functionality of the system. We will measure the percentage time for which the system is down on a monthly basis (numerator: number of times (in minutes) when the system is down; denominator total active time in a month. Down time defined as $30 \mathrm{~min}$ of hanging and active time as time without hitches), proportion of CHVs effectively using the decision support system (within the system, on a quarterly basis we will be able to generate reports on decisions, correct or otherwise, made by the CHVs. These will also be compared with the control site that will only have a paper-based system of data collection) and the experiences of the CHVs and the mothers with the mobile-based system. These will be compared with the investment.

To answer the question on effect of the system on use of services, the data above will be triangulated with data from the 'mPAMANECH application which already has an integrated data collection module', the data generated by the CHVs and participating health facilities will be retrieved, cleaned and analysed to derive estimates of the main outcome of interest-correct referral practices. In addition, these data will be triangulated with other sources such as the CHV monthly reports and health facility HMIS. A system of random numbers generated using STATA will be used to select the respondents based on a sampling frame that is going to be informed by an updated household register in the selected community units.

\section{Qualitative study}

We will use focus group discussions (FGDs) and in-depth interviews among the direct project beneficiaries and CHVs, and key informant interviews with key actors (SCHMTs, health providers, CHVs and community leaders). Participants will be purposively selected to represent the different stakeholders as well as different health service user categories (users and non-users). 
Data from the quantitative survey will be used to identify women who have or not used specific MNH services, and these will be approached to participate in the focus group discussions or in-depth interviews. Other respondents will be identified based on their position in the community and their role in the project.

\section{Data collection, management and analysis Data collection}

We will conduct a baseline survey and an end line at least 1 year after the full implementation of the intervention. The control group will be assessed at the same time as the surveys are being conducted in the intervention group. To strengthen the case for causal inference, we will also do a systematic monitoring and documentation of the intervention based on our theory of change. The monitoring and documentation will also capture any other contextual factors that may influence the same outcomes as our intervention. The effects and impact of the project will be determined by triangulating data and information from different sources, examining trends where possible and trying to find and support explanations for the observed findings.

Our key data sources will be the following: household surveys, routine HMIS data and qualitative assessments (interviews and FGDs).

We will conduct simple and multiple logistic regression analyses comparing differences in the proportions of women in reproductive age and children under five at baseline and end line for variables like antenatal coverage, vaccination coverage, skilled birth attendance, among others, (see figure 1 for key indicators). These analyses will control for any differences in the samples (if any) at the two time points as well as the contribution of contextual factors that may have occurred in the course of the implementation.

mPAMANECH data: Descriptive data in terms of referrals by diagnostic decisions, among others, will be summarised using means and SD for the parametric continuous data and medians with interquartile ranges for the non-parametric data. Categorical data such as referrals by age and gender will be summarised using proportions and percentages.

Qualitative data: Qualitative data will be transcribed and saved in Word format. Transcribed word files will be imported into NVivo software (QSR International) for coding and further analysis. Analysis across all transcripts will be conducted using a constant comparative method to identify themes and their repetitions and variations. The analysis will also aim to identify changes (if any) in various indicators that could be attributed to the intervention. Figure 5 shows the study's timeline.

\section{ETHICS AND DISSEMINATION}

The study was reviewed internally by APHRC's internal scientific review committee. Ethical approval for the project was obtained from the African Medical Research

\begin{tabular}{|c|c|c|c|c|c|c|c|}
\hline \multirow[b]{2}{*}{ Activitiy } & \multicolumn{3}{|c|}{2016} & \multicolumn{4}{|c|}{2017} \\
\hline & Q1 & Q2 & Q3 & Q4 & Q5 & Q6 & Q7 \\
\hline \multicolumn{8}{|l|}{ Inception period } \\
\hline \multicolumn{8}{|l|}{ Seek ethical approval } \\
\hline \multicolumn{8}{|l|}{ Recruit and train field team } \\
\hline \multicolumn{8}{|l|}{ Conduct baseline } \\
\hline \multicolumn{8}{|l|}{ Conduct endline } \\
\hline \multicolumn{8}{|l|}{ Data cleaning } \\
\hline \multicolumn{8}{|l|}{ Data analysis and intepretation } \\
\hline \multicolumn{8}{|l|}{ Scientific writeup on baseline } \\
\hline \multicolumn{8}{|l|}{ Scientific writeup on endline } \\
\hline \multicolumn{8}{|c|}{ Developing decision support system } \\
\hline \multicolumn{8}{|c|}{$\begin{array}{l}\text { Training community health assistants } \\
\text { (CHAs) }\end{array}$} \\
\hline $\begin{array}{l}\text { Training sub-county health } \\
\text { mangment teams (sCHMTs) }\end{array}$ & & & & & & & \\
\hline
\end{tabular}

Figure 5 Project timeline. CHV, community health volunteer; CHW, community health worker,sCHMT subCounty Health Management Teams

Fund's Ethical and Scientific Review Committee (AMREFESRC P279/2016). The research team has undergone the National Institute for Health (NIH) training on protecting human research participants. We shall seek informed consent from all study participants. All survey data will be collected in privacy and will be treated with confidentiality. It will be made clear to participants from the onset that participation in the study is voluntary and that they may choose to withdraw from the study at any time and for whatever reasons without fear of penalty. The mPAMANECH datasets will be available to users (CHVs, clinicians, CHEWs, among others) based on assigned, but limited, rights to protect the identity of study participants. Only the core research team will have access to all of this information. The entire dataset will be stored in the APHRC server.

The potential risks for study participants are limited to (1) data breaches, (2) time spent on the interview and (3) discomfort experienced due to particular questions that respondents may perceive as upsetting or personal. These potential risks will be carefully explained to each participant during the process of obtaining informed consent.

For the data collected on the phones and computers, once the data are saved, they are relayed in real time to the APHRC server and no information is saved on the phone or the computer. The users will only be able to see summary data, without the participants' details. To further minimise the risk attached to data breaches following the loss of a smartphone, each phone will have a four-digit pass code to enable the CHVs log into the phone. This code will become active after $3 \mathrm{~min}$ on non-use. The third level of security is the username and password on each device.

\section{Plan for communicating findings of the study}

Results obtained through this study will be made available to those who contributed information; the communities of Kamukunji and Embakasi. In addition: 
1. The study team will conduct one major dissemination workshop in Kenya to share research findings with development partners, local nongovernmental organisations, healthcare providers, activists, media houses, health professional societies and policy-makers.

2. Findings of the study will be shared at local and international conferences with researchers and policymakers.

3. We also expect to publish three peer-reviewed articles in Open Access scientific journals.

4. The Policy Engagement and Communications unit at APHRC will also use the findings of this study to produce policy briefs, fact sheets and working papers, as well as newsletters, which will be shared with a wide range of stakeholders.

5. The findings of the study will also be shared through APHRC's website.

\section{DISCUSSION}

Maternal and neonatal health are both national and global priorities given that maternal and neonatal health indicators have not appreciably improved over the millennium development goals life span. They remain aspirations in the sustainable development agenda. CHVs are a critical component of the health workforce bridging the gap between communities and the formal health system. They not only improve coverage and access to basic health services but are also usually available in locations where and at times when formal health services are unavailable or inadequate. Quality healthcare is largely supported by quality data. Kenya still faces challenges with its health management information system. Inaccurate data as a result of poor documentation impair the country's planning processes. This project accords APHRC and its partners an opportunity to use funds that have been identified to respond to Kenya's needs; to improve maternal and newborn health.

The next steps of the project if the experiments under this grant are successful will include sharing the findings with the Nairobi City County and the funder and agreeing on the next phase of implementation. The Ministry of Health, Nairobi, is keen to roll out the system county-wide subject to availability of funding. The project team will work with the consortium to generate evidence to support decision-making at whatever stage of implementation. These may include effectiveness in reducing adverse maternal and neonatal outcomes at national level, cost-effectiveness, process issues such as CHV motivation and efficiency among others.

Potential impediments to the intervention include possible technology failure, loss of equipment (phones) and CHV turnover; all of which could require revisions and adaptations in the work plan (eg, quality assurance, insurance and training). A potential harmful unintended consequence stems from the fact that CHVs are not medically trained and as such could make a decision to treat at home rather than refer (even when the system advises otherwise) with possible negative health consequences for the mothers and their newborns. However, since the system allows health workers to access these data, they will be in charge of following up on such cases to avoid unnecessary delays and such unintended consequences.

\section{CONCLUSION}

The study outlined in this protocol will assess the added value of using a CHV decision-support module of mPAMANECH in reducing maternal complications and newborn deaths in the informal settlements of Nairobi, Kenya. The study's findings will contribute to the body of knowledge on the effectiveness of mHealth innovations in improving maternal, newborn and child health services and outcomes in urban informal settlements. Successful execution of the protocol will generate evidence on the effectiveness, if any, of the system. Based on the positive lessons learnt, this project will provide a system for adoption by the local and central governments for resource-limited communities.

Contributors PB, CK, EK and AKZ conceived the project and its design and participated in refining the manuscript. MO contributed to the refining of the design. PB drafted the manuscript. All authors read and approved the final manuscript.

Funding This project is supported by the County Innovation Challenge Fund for Kenya (GR: CICF-INN-R2-088). The funder had no role in the decision to write and publish this protocol.

Competing interests None declared.

Ethics approval African Medical and Research Foundation (AMREF) ESRC.

Provenance and peer review Not commissioned; externally peer reviewed.

Open Access This is an Open Access article distributed in accordance with the Creative Commons Attribution Non Commercial (CC BY-NC 4.0) license, which permits others to distribute, remix, adapt, build upon this work non-commercially, and license their derivative works on different terms, provided the original work is properly cited and the use is non-commercial. See: http://creativecommons.org/ licenses/by-nc/4.0/

(c) Article author(s) (or their employer(s) unless otherwise stated in the text of the article) 2017. All rights reserved. No commercial use is permitted unless otherwise expressly granted.

\section{REFERENCES}

1. Say L, Chou D, Gemmill A, et al. Global causes of maternal death: a WHO systematic analysis. Lancet Glob Health 2014;2:e323-33.

2. Liu L, Johnson HL, Cousens S, et al. Global, regional, and national causes of child mortality: an updated systematic analysis for 2010 with time trends since 2000. Lancet 2012;379:2151-61.

3. WHO. Neonatal and perinatal mortality: country, regional and global estimates. Geneva: World Health Organization, 2006.

4. Jehan I, Harris H, Salat S, et al. Neonatal mortality, risk factors and causes: a prospective population-based cohort study in urban Pakistan. Bull World Health Organ 2009;87:130-8.

5. KNBS, ICFMacro. Kenya demographic and health survey 2008-09. Calverton, Maryland: Kenya National Bureau of Statistics (KNBS) and ICF Macro, 2010.

6. KNBS. Kenya demographic and health survey 2014. Nairobi, Kenya: Kenya National Bureau of Statistics (KNBS), 2015.

7. Lehmann U, Sanders D. Community health workers: what do we know about them the state of the evidence on programmes, activities, costs and impact on health outcomes of using community health workers. Geneva: World Health Organization, 2007:1-42.

8. Christopher JB, Le May A, Lewin S, et al. Thirty years after AlmaAta: a systematic review of the impact of community health workers delivering curative interventions against malaria, pneumonia and 
diarrhoea on child mortality and morbidity in sub-Saharan Africa. Hum Resour Health 2011;9:27.

9. Gilmore B, McAuliffe E. Effectiveness of community health workers delivering preventive interventions for maternal and child health in low- and middle-income countries: a systematic review. BMC Public Health 2013;13:847.

10. Mumbo HM, et al. Uptake of task shifting as a community strategy in Kenya. European Scientific Journal 2013;9:14.

11. Berner ES. Clinical decision support systems. New York: Springer, 2007.

12. Kawamoto K, Houlihan CA, Balas EA, et al. Improving clinical practice using clinical decision support systems: a systematic review of trials to identify features critical to success. BMJ 2005;330:765.

13. Garg AX, Adhikari NK, McDonald H, et al. Effects of computerized clinical decision support systems on practitioner performance and patient outcomes: a systematic review. JAMA 2005;293:1223-38.

14. Bates DW, Kuperman GJ, Wang S, et al. Ten commandments for effective clinical decision support: making the practice of evidence-based medicine a reality. J Am Med Inform Assoc 2003;10:523-30.

15. Agarwal S, LeFevre AE, Lee J, et al. Guidelines for reporting of health interventions using mobile phones: mobile health (mHealth) evidence reporting and assessment (mERA) checklist. BMJ 2016;352:i1174

16. Blaya JA, Fraser HS, Holt B. E-health technologies show promise in developing countries. Health Aff 2010;29:244-51.

17. Vodafone. Connected worker: how mobile technology can improve working life in emerging economies. 2015. http://www.vodafone. $\mathrm{com} /$ content/dam/sustainability/pdfs/vodafone_connected_worker. pdf

18. Lucas $\mathrm{H}$. Information and communications technology for future health systems in developing countries. Soc Sci Med 2008;66:2122-32.

19. Lund S, Rasch V, Hemed M, et al. Mobile phone intervention reduces perinatal mortality in zanzibar: secondary outcomes of a cluster randomized controlled trial. JMIR Mhealth Uhealth 2014;2:e15.
20. Noordam AC, Kuepper BM, Stekelenburg J, et al. Improvement of maternal health services through the use of mobile phones. Trop Med Int Health 2011;16:622-6.

21. Free C, Phillips G, Galli L, et al. The effectiveness of mobile-health technology-based health behaviour change or disease management interventions for health care consumers: a systematic review. PLOS Med 2013;10:e1001362.

22. Braun R, Catalani C, Wimbush J, et al. Community health workers and mobile technology: a systematic review of the literature. PLOS One 2013;8:e65772.

23. Källander K, Tibenderana JK, Akpogheneta OJ, et al. Mobile health (mHealth) approaches and lessons for increased performance and retention of community health workers in low- and middle-income countries: a review. J Med Internet Res 2013;15:e17.

24. Bakibinga P, Ettarh R, Ziraba AK, et al. The effect of enhanced public-private partnerships on Maternal, Newborn and child Health Services and outcomes in Nairobi-Kenya: the PAMANECH quasi-experimental research protocol. BMJ Open 2014;4:e006608

25. APHRC. Harnessing Public-Private Partnership to Improve Maternal, Newborn and Child Healthservices and Outcomes in Nairobi Slum Settlement: End of Grant Report. Nairobi, Kenya: African Population \& Health Research Center (APHRC). 2016.

26. Baqui AH, Arifeen SE, Williams EK, et al. Effectiveness of homebased management of newborn infections by community health workers in rural Bangladesh. Pediatr Infect Dis J 2009;28:304-10.

27. Waiswa P, et al. Effect of the Uganda Newborn Study on careseeking and care practices: a cluster-randomised controlled trial. Glob Health Action 2014;7:24584.

28. MOH. MOHM.o.H. Division of Community Health Services, Kenya, ed. Community Health Volunteers (CHVs): Basic modules handbook. Nairobi, Kenya, 2013.

29. RedCross-Kenya. Training CHWs in Kenya - Lessons Learned, 2014. (accessed 25 Aug 2016).

30. WHO. Mother and Child Card: danger signs, 2012. (accessed 13 Aug 2016). 